\title{
Further identification of ROSAT all-sky survey sources in Orion`
}

\author{
A. Frasca ${ }^{1}$, J. M. Alcalá ${ }^{2}$, E. Covino ${ }^{2}$, S. Catalano ${ }^{1}$, E. Marilli ${ }^{1}$, and R. Paladino ${ }^{2}$ \\ 1 INAF, Osservatorio Astrofisico di Catania, via S. Sofia 78, 95123 Catania, Italy \\ 2 INAF, Osservatorio Astronomico di Capodimonte, via Moiariello 16, 80131 Napoli, Italy
}

Received 17 February 2003 / Accepted 24 April 2003

\begin{abstract}
We report on the identification of 22 ROSAT All-Sky Survey (RASS) X-ray sources distributed in the general direction of the Orion star-forming region. The X-ray sample contains sources from the ROSAT bright source catalogue and from previous detections. The optical identifications are based on intermediate-resolution spectroscopy and $U B V$ Johnson photometry using a $1 \mathrm{~m}$-class telescope. The strengths of the $\mathrm{H} \alpha, \mathrm{NaID}_{2}$ and lithium lines for the stellar counterparts are evaluated applying the spectral subtraction technique, using templates of the same spectral type. Radial velocities of the optical counterparts are also reported. Thirteen of the optical counterparts show the lithium absorption line in their spectra and have radial velocities consistent with the Orion star forming region. Four of these objects can be classified as new bona-fide T Tauri stars.
\end{abstract}

Key words. X-ray: stars - stars: pre-main-sequence - stars: low-mass, brown dwarfs - stars: activity -

ISM: individual objects: Orion - stars: formation

\section{Introduction}

The observations with the Einstein X-ray satellite in starforming regions (SFRs) showed that many low-mass pre-main sequence (PMS) stars are strong X-ray emitters (Walter 1986). More recently, several investigations with the ROSAT satellite, mainly based on the ROSAT All-Sky Survey (RASS) X-ray data, revealed many new low-mass PMS stars, spread not only in the star forming clouds, but also distributed in the surroundings of star formation complexes (see Feigelson \& Montmerle 1999 and Walter et al. 2000, and references therein). Most of these objects belong to the weak T Tauri star (WTTS) class.

Subsequent investigations based on high-resolution spectroscopy demonstrated that most of these stars are indeed lowmass PMS stars (Covino et al. 1997; Wichmann et al. 1999; Alcalá et al. 2000). Feigelson (1996) suggested that many of the WTTS distributed in SFRs were formed in cloudlets which dissipated immediately after star formation, while others proposed that a considerable number of these stars may be members of the Gould Belt (Wichmann et al. 1997; Guillout et al. 1998).

Astrometric data are now available for many of the distributed WTTS, as entries of the Hipparcos and Tycho catalogues. Such data indicate probable membership for several of the distributed WTTS to the SFRs on which they are projected

\footnotetext{
Send offprint requests to: A. Frasca,

e-mail: afr@sunct.ct.astro.it

* Based on observations collected at Catania Astrophysical Observatory, Italy.
}

(cf. Bertout et al. 1999), and the results of proper motion studies (cf. Frink et al. 1998) appear to be in line with the simulations by Feigelson (1996) of the low-mass star formation in small cloudlets.

The identification of RASS sources in SFRs is still providing new relevant discoveries. For instance, the recent spectroscopic follow-up in Sco-Cen by Mamajek et al. (2002), based on the correlation of the astrometric ACT Catalogue and Tycho Reference Catalogue (TRC) with the X-ray counterparts in the RASS bright source catalogue, yielded a success rate higher than $70 \%$ in selecting PM stars.

A particularly interesting SFR is the Orion complex in which at least three regions with different age and location, connected to the OB associations Ori 1a, 1b, and 1c, can be distinguished (Blaauw 1991). This suggests that the star formation rate in younger parts of the complex has been likely influenced by supernova explosions in the relatively older regions, like Ori OB1a.

Based on the X-ray properties of sources discovered in the ROSAT All-Sky Survey, Sterzik et al. (1995) performed an extensive search for low-mass PMS stars over a region of $710 \mathrm{deg}^{2}$ around the Orion complex, and selected a sample of about 1000 PMS candidates. Furthermore, they identified four $\mathrm{X}$-ray source density enhancements associated with Ori OB1a, $\lambda$ Ori, OB1b, and OB1c regions.

More than 1000 PMS candidates have also been detected as infrared variables in a $0.84 \times 6$ square degrees region around the Orion Trapezium cluster by Carpenter et al. (2001) by means of infrared photometry from the 2MASS survey. 
The observed variability on several time scales is ascribed to different physical mechanisms and mainly due to rotational modulation of cool and hot starspots, disks and changes in the accretion rate.

In this paper, we report on further optical identifications of ROSAT All-Sky Survey (RASS) X-ray sources in the general direction of the Orion SFR. The identifications are based on medium-resolution optical spectroscopy and $U B V$ photoelectric photometry using a $1 \mathrm{~m}$-class telescope. For this reason, the investigated X-ray sample is limited to 22 RASS sources with most probable optical counterparts brighter than about $V=12 \mathrm{mag}$. The studied sample, listed in Table 1, includes sources from the RASS bright source catalogue, as well as from the list of 820 RASS sources detected by Alcalá et al. (1996).

In order to test the reliability of the optical identifications, as well as the detection and strength of the lithium absorption line, the two X-ray sources RX J0502.4-0744 and RX J0530.1+0041, already known to be WTTS (Alcalá et al. 2000), were included in our sample as test targets.

In Sect. 2 we present the spectroscopic identifications and photometric observations, as well as describe the data reduction. In Sect. 3 the main results from our photometric and spectroscopic data are discussed. The spatial distribution and kinematical properties of the PMS stars in Orion are examined in Sect. 4, and in Sect. 5 the HR diagram of the stars with detectable lithium is presented. Finally, our conclusions are presented in Sect. 6.

\section{Observations}

The sample of $22 \mathrm{X}$-ray sources studied here represents an extension of previous identification works (e.g. Alcalá et al. 1996), although limited to optical counterparts brighter than about $V=12 \mathrm{~m} 0$. Using the USNO catalogue, we have selected all the stars brighter than $R=12$. 0 in the area under study. A cross-correlation wih the RASS bright source catalogue yields 109 matches, using a correlation radius of $40{ }^{\prime \prime}$. (see Neuhäuser et al. 1995). Hence, our sample represents about $20 \%$ of the bright X-ray star sample in the region.

In the study by Sterzik et al. (1995) of RASS sources in Orion it was concluded that some thousand sources, out of 2257, are expected to have pre-main sequence star counterparts, which represent about $44 \%$ of their studied sample. Extrapolating this success rate to our sample, it would be expected to find some 10 PMS counterparts. The number would be slightly smaller because the success rate decreases for bright objects.

\subsection{Spectroscopy}

The spectroscopic observations were carried out between December 6th and 18th, 2000 at M.G. Fracastoro station (Serra La Nave, Mt. Etna, Italy) of the Catania Astrophysical Observatory with the REOSC spectrograph fed by the 91-cm telescope through an UV-NIR fiber of $200 \mu \mathrm{m}$ core diameter. For the spectroscopic identifications, the brightest stars closest to the RASS error box centers (listed in Table 1) were observed. The image-scale at the focal plane of the Cassegrain telescope $\left(14.4^{\prime \prime} / \mathrm{mm}\right)$, combined with the microlens adapter, makes the fiber able to collect light from about $10^{\prime \prime}$ on the sky. The observations were obtained using the low-dispersion configuration which employs the 900-lines/mm Milton-Roy echellette grating as dispersing element and yields a linear dispersion of $37 \AA / \mathrm{mm}$.

The spectra were acquired with a $1024 \times 1024$ SITe thinned back-illuminated CCD of $24 \times 24 \mu \mathrm{m}$ pixel size, yielding a spectral range from $5860 \AA$ to $6780 \AA$, and a dispersion of $0.90 \AA$ per pixel. For all observing runs the width of the slit was set to $400 \mu \mathrm{m}$, projected onto 2.4 pixels on the detector, as evaluated from the $F W H M$ of the emission lines of Th-Ar calibration lamp, providing a spectral resolution of about $2 \AA$. Such resolution is sufficient for the identification of the $\operatorname{Li} \lambda 6708 \AA$ absorption line, used as the primary youth indicator for stars with spectral types later than about G5; the line is well resolved from the $\mathrm{Ca} \lambda 6718 \AA$ absorption line and measurements of the Li equivalent width can be performed with an average error of less than about $50 \mathrm{~m} \AA$, depending on the $\mathrm{S} / \mathrm{N}$ ratio of the spectrum and spectral type (see Covino et al. 1997). Indeed the lithium absorption line was clearly detected in the two previously known WTTS RX J0502.4-0744 and RX J0530.1+0041.

Multiple observations were obtained for more than one half of the optical counterparts. In addition to our targets, we observed 33 standard stars, both giants and dwarfs spanning a wide range in spectral type, to be used as templates for the spectral type determinations. Some of these standard stars are also radial velocity standards and were used as templates for the cross-correlation analysis (see Sect. 3.2).

Typical exposure times for the spectroscopic observations span from 1800 to $3600 \mathrm{~s}$, according to their magnitude, sky transparency and seeing conditions. The signal-to-noise ratio $(\mathrm{S} / \mathrm{N})$ achieved was between 20 and 100. The less exposed spectra have, in any case, a $\mathrm{S} / \mathrm{N}$ ratio sufficient to perform a good cross-correlation analysis and to extract information about the $\mathrm{H} \alpha$-line emission or filling-in. The LiI $\lambda 6708$ line identification is less secure in the worst cases.

The data reduction, including bias, scattered light and flat field corrections, was performed using an IDL procedure properly written. Cosmic-ray hits were also automatically eliminated by comparing the flux distribution of the targets across the dispersion, at each wavelength, with that produced by spectra of bright stars acquired in the same night with low exposure times $(\leq 1-2 \mathrm{~min})$, which are almost free from cosmics.

The wavelength calibration was performed using the IRAF ${ }^{1}$ tasks IDENTIFY and REFSPEC and the spectra of the Th-Ar lamp.

For the subsequent analysis, the spectra have been normalized to the local continuum by fitting a low-order polynomial to the line-free spectral regions by means of the CONTINUUM task under IRAF package.

\footnotetext{
${ }^{1}$ IRAF is distributed by the National Optical Astronomy Observatory, which is operated by the Association of the Universities for Research in Astronomy, inc. (AURA) under cooperative agreement with the National Science Foundation.
} 
Table 1. Investigated RASS sources in Orion. Column 2: X-ray source designation; Cols. 3 and 4: X-ray source coordinates; Col. 5: ROSAT broad-band count rate; Col. 6: X-ray exposure time; Col. 7: other identification/designation.

\begin{tabular}{|c|c|c|c|c|c|c|}
\hline$\#$ & $\mathrm{RXJ}$ & $\begin{array}{c}\alpha(2000) \\
\text { h m s }\end{array}$ & $\begin{array}{c}\delta(2000) \\
\circ, \prime \prime\end{array}$ & $\mathrm{cts} / \mathrm{s}^{a}$ & $\begin{array}{l}\text { Exp. Time } \\
\text { (s) }\end{array}$ & Ident. \\
\hline 1 & RX J0500.7-0456 & 050041.0 & -045618 & $0.087 \pm 0.020$ & 398.5 & SD-05 1122 \\
\hline 2 & RX J0502.0+0959 & 050158.9 & +095900 & $0.931 \pm 0.055$ & 437.0 & HIC 23418, RBTS 285 \\
\hline 3 & RX J0502.4-0744 & 050221.1 & -074403 & $0.050 \pm 0.015$ & 414.5 & \\
\hline 4 & RX J0503.1+0548 & 050306.2 & +054848 & $0.051 \pm 0.015$ & 426.6 & GSC 00110-01074 \\
\hline 5 & 1RXS J050315.2+084101 & 050315.1 & +084107 & $0.065 \pm 0.016$ & 451.6 & \\
\hline 6 & RX J0510.7-0316 & 051040.5 & -031631 & $0.042 \pm 0.019$ & 422.0 & GSC04755-00873 \\
\hline 7 & RX J0511.7-0809 & 051141.8 & -080912 & $0.048 \pm 0.027$ & 178.0 & IRAS 05092-0814? \\
\hline 8 & 1RXS J051523.9-091234 & 051523.8 & -091233 & $0.072 \pm 0.021$ & 412.4 & \\
\hline 9 & 1RXS J051631.5-080510 & 051631.2 & -080507 & $0.049 \pm 0.020$ & 409.7 & \\
\hline 10 & RX J0517.0-0907 & 051659.8 & -090726 & $0.034 \pm 0.017$ & 409.4 & BD-09 1112,SAO 131944 \\
\hline 11 & RX J0517.9-0708 & 051756.4 & -070824 & $0.123 \pm 0.048$ & 173.4 & \\
\hline 12 & RX J0523.1-0741 & 052303.1 & -074150 & $0.032 \pm 0.015$ & 386.9 & GSC 05332-00283 \\
\hline 13 & 1RXS J052345.0-075343 & 052344.7 & -075349 & $0.145 \pm 0.029$ & 365.8 & \\
\hline 14 & RX J0524.4-0640 & 052423.6 & -064004 & $0.025 \pm 0.016$ & 330.5 & \\
\hline 15 & RX J0529.8-0102 & 052950.3 & -010216 & $0.032 \pm 0.014$ & 473.7 & HD 290521 \\
\hline 16 & RX J0530.1+0041 & 053005.3 & +004118 & $0.193 \pm 0.030$ & 495.5 & HD 290477 \\
\hline 17 & 1RXS J053114.3-092237 & 053114.3 & -092237 & $0.071 \pm 0.019$ & 446.0 & \\
\hline 18 & RX J0533.7-0521 & 053343.5 & -052122 & $0.036 \pm 0.015$ & 487.6 & V730Ori \\
\hline 19 & RX J0535.0-0618 & 053457.5 & -061828 & $0.064 \pm 0.019$ & 473.0 & 2E $0532.5-0620$ \\
\hline 20 & RX J0536.1-0541 & 053605.8 & -054131 & $0.064 \pm 0.020$ & 489.4 & Parenago 2328 \\
\hline 21 & RX J0551.5-0628 & 055130.4 & -062857 & $0.017 \pm 0.010$ & 534.6 & \\
\hline 22 & 1RXS J055841.6-031123 & 055841.4 & -031122 & $0.056 \pm 0.018$ & 497.5 & \\
\hline
\end{tabular}

a A rough estimate of the observed X-ray flux can be determined assuming a mean counts-to-energy conversion factor of $1.22 \times$ $10^{-11} \mathrm{erg} \mathrm{cm}^{-2} \mathrm{~s}^{-1}$ count $^{-1}$.

\subsection{Photometry}

The photometric observations were carried out from November 14th to 16th 2001 and from December 18th to 19th 2001, in the Johnson $U B V$ standard system, also with the $91-\mathrm{cm}$ telescope, at Serra La Nave. The observations were performed with a photon-counting photometer equipped with an EMI 9893QA/350 photomultiplier, cooled to $-15{ }^{\circ} \mathrm{C}$. The dark noise of the detector, operated at this temperature, is about 1 photon/s.

Field stars of known magnitudes and color indices were observed nightly to transform the instrumental magnitudes to the Johnson $U B V$ photometric system. Fourteen stars angularly close to our targets (within 5 degrees) and with known $U B V$ magnitudes taken from the General Catalogue of Photometric Data (GPCD, Mermilliod et al. 1997) were observed with the same instrument set-up in between the targets observations. These "local" standard stars and the optical counterparts to the RASS sources were grouped in seven fields according to their proximity on the sky (I-VII in Tables 2 and 3) and the magnitudes and colors of the local standards (Table 2) were used to determine photometric "zero points" for the instrumental $U V B$ magnitudes. Additionally, several standard stars, selected from the list of Landolt (1992), were also observed during the run for the determination of the transformation coefficients to the Johnson standard system as well as to check the magnitudes and colors of the comparison stars listed in Table 2.

A typical observation consisted of several cycles (from 2 to 5, depending on the star brightness) of integrations of 10,5, $5 \mathrm{~s}$, in the $U, B$ and $V$ filter, respectively. A $21^{\prime \prime}$ diaphragm was used. The data were reduced by means of the photometric data reduction package PHOT designed for photoelectric photometry of Catania Observatory (Lo Presti \& Marilli 1993). Seasonal mean extinction coefficient for Serra La Nave Observatory were adopted for the atmospheric extinction correction.

RX J0530.1+0041 was observed from December 1st, 2000 to February 26th, 2001 in the $B$ and $V$ filters only, during a campaign aimed to study rotational modulation induced by starspot activity in PMS.

\section{Results}

\subsection{UBV photometry}

The resulting $U B V$ photometry of the program stars is presented in Table 3. In this table, the coordinates of the optical counterparts, from the USNO-B1 catalogue 
Table 2. Magnitudes and colors of the comparison and standard stars.

\begin{tabular}{lrrcc}
\hline \hline Name & $V$ & $B-V$ & $U-B$ & Field \\
\hline HD 31799 & 8.06 & +0.06 & +0.16 & I \\
HD 32925 & 9.21 & +0.33 & +0.05 & I \\
HD 34639 & 9.61 & +0.00 & -0.34 & II \\
HD 34814 & 9.10 & +0.00 & -0.10 & II \\
HD 35718 & 9.02 & +0.04 & -0.04 & III \\
HD 35225 & 8.59 & +0.21 & +0.09 & III \\
HD 290517 & 9.00 & -0.05 & -0.29 & IV \\
HD 290516 & 9.47 & +0.03 & -0.14 & IV \\
HD 31766 & 8.42 & +0.08 & +0.01 & V \\
HD 32420 & 9.57 & +0.07 & +0.01 & V \\
HD 36513 & 9.50 & +0.03 & -0.11 & VI \\
HD 36867 & 9.30 & -0.02 & -0.16 & VI \\
HD 39161 & 8.94 & +0.09 & +0.05 & VII \\
HD 40534 & 9.06 & +0.01 & -0.27 & VII \\
\hline
\end{tabular}

(Monet et al. 2003), are given. The photometric errors, estimated from measurements of standard stars with comparable brightness to the program stars, are $\sigma_{V}=0.01, \sigma_{B-V}=0.02$, $\sigma_{U-B}=0.02$.

Since we have several observations for each star on the same or in different nights, we have listed the average $V$ magnitudes and colors, with the standard deviation in brackets.

Following the method described by Fitzgerald (1970) and Crawford \& Mandwewala (1976), we have evaluated the reddening parameters $E_{B-V}$ and $A_{V}$, also listed in Table 3, from the reddening lines in the $(B-V)-(U-B)$ diagram (see Fig. 1). Three stars, namely RX J0502.0+0959, RX J0533.7-0521 and RX J0551.5-0628 lie off the main sequence in the diagram $(B-V)-(U-B)$. The first star, RX J0502.0+0959, a very active dMe field star projected against the Orion SFR, displays a high $U-B$ excess, which is probably due to facular contribution at UV wavelengths (see e.g. Amado \& Byrne 1997), while RX J0533.7-0521 and RX J0551.5-0628 are embedded in dense nebular regions whose diffused light affects their colors. For these three stars, no de-reddening correction could be applied. The spectra of two of these stars (RX J0502.0+0959 and RX J0533.7-0521) are shown in Fig. 2.

\subsection{Radial velocities}

The radial velocity $(R V)$ measurements of our targets (reported in Table 4) were obtained by means of the cross-correlation technique, using the IRAF task FXCOR. The radial velocity standard stars $\alpha$ Ari and 54 Aql, whose radial velocities were taken from Evans (1967) and Duflot et al. (1995), were used as templates for the cross-correlation analysis. The $\mathrm{H} \alpha$ line, which may be contaminated by chromospheric emission, was excluded from the computation of the cross-correlation function (CCF). In order to better evaluate the centroids of the CCF peaks, we adopted Gaussian fits.

The standard errors $\sigma_{\mathrm{i}}$ in the $R V$ values are computed from the FXCOR task according to the fitted peak height and the antisymmetric noise as described by Tonry \& Davis (1979). These

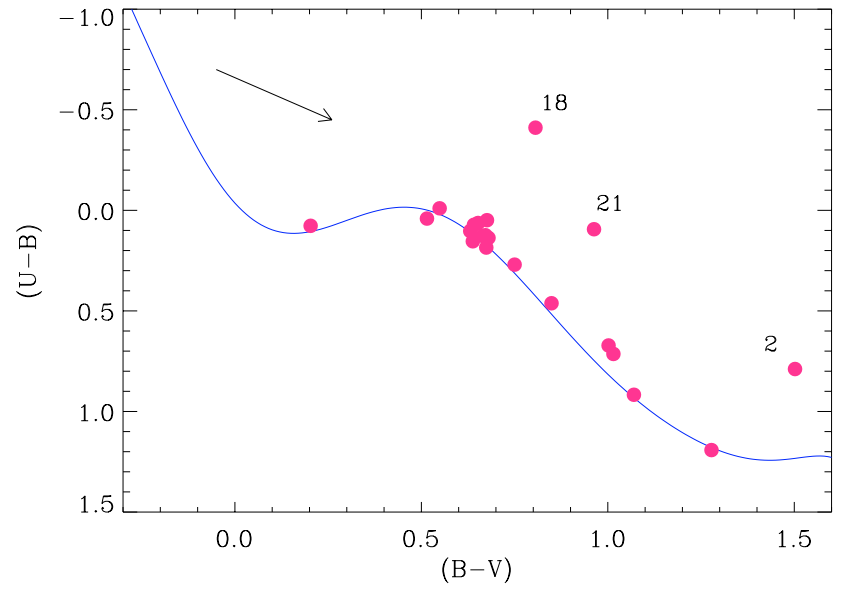

Fig. 1. $(B-V)-(U-B)$ color-color diagram for the stars in our sample. The solid line represents the intrinsic colors for dwarf stars from Fitzgerald (1970) and the arrow indicates the reddening vector.

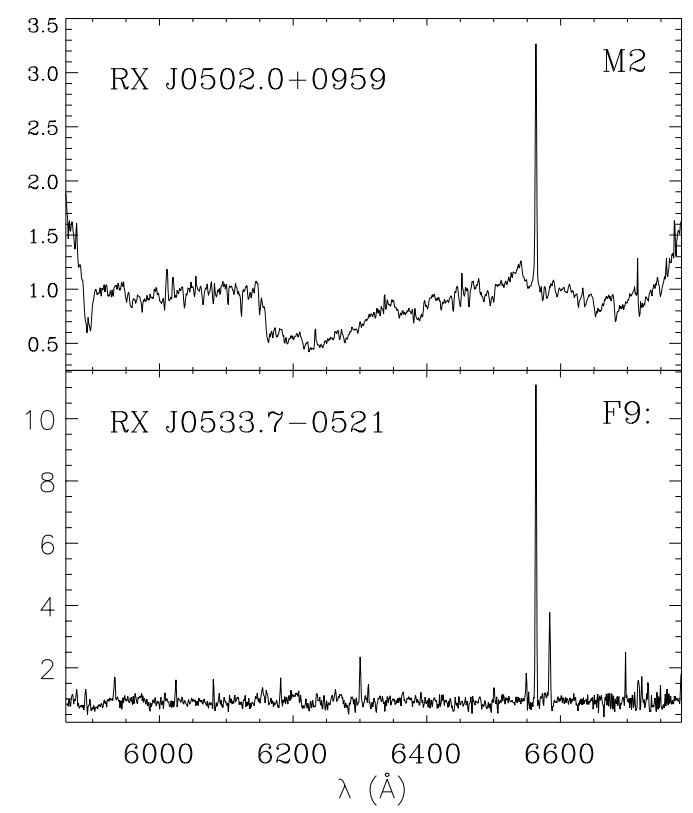

Fig. 2. Spectra of two stars in our sample. Upper panel: the very active field dMe star RX J0502.0+0959; lower panel: a PMS star embedded into a dense nebula, which gives rise to the strong emission lines.

errors are in the range 5-20 $\mathrm{km} \mathrm{s}^{-1}$, with some exception for the stars with low $\mathrm{S} / \mathrm{N}$, for which a larger error has been found. For the well exposed spectra the precision on $R V$ measurements is mainly limited by the spectral resolution (that prevents a precise $R V$ analysis), but is sufficiently high to provide an indication of the spatial motion, to be compared with that of the Orion SFR, and to detect any $R V$ variation larger than $\sim 10 \mathrm{~km} \mathrm{~s}^{-1}$, indicative of binarity.

\subsection{Spectral type determination}

Spectral types were determined by comparing the spectrum of each target with a grid of spectra of bright standard stars (from F8 to M3) observed during the campaign with the same instrumental setup. For this purpose, we have developed an IDL 
Table 3. Magnitudes and colors of targets.

\begin{tabular}{|c|c|c|c|c|c|c|c|c|c|}
\hline $\bar{H}$ & RXJ & $\alpha(2000)^{a}$ & $\delta(2000)^{a}$ & Field & $\overline{\bar{V}}$ & $B-V$ & $\overline{U-B}$ & $\begin{array}{l}\text { N. of } \\
\text { meas. }\end{array}$ & $\overline{E E_{B-V}}$ \\
\hline 1 & RX J0500.7-0456 & 050040.88 & -045616.3 & $\bar{I}$ & $9.94(.01)$ & $0.52(.01)$ & $0.04(.02)$ & 6 & $\overline{0.06}$ \\
\hline 2 & RX J0502.0+0959 & 050158.80 & +095859.9 & $\mathrm{V}$ & $11.50(.03)$ & $1.50(.01)$ & $0.79(.11)$ & 4 & - \\
\hline 3 & RX J0502.4-0744 & 050220.86 & -074410.0 & I & $11.23(.02)$ & $0.68(.01)$ & $0.14(.02)$ & 4 & 0.03 \\
\hline 4 & RX J0503.1+0548 & 050305.89 & +054840.2 & $\mathrm{~V}$ & $10.68(.01)$ & $0.65(.01)$ & $0.06(.01)$ & 5 & 0.07 \\
\hline 5 & 1RXS J050315.2+084101 & 050314.69 & +084041.9 & $\mathrm{~V}$ & $11.50(.03)$ & $1.07(.02)$ & $0.92(.02)$ & 4 & 0.03 \\
\hline 6 & RX J0510.7-0316 & 051040.51 & -031641.6 & I & $11.38(.03)$ & $0.63(.01)$ & $0.10(.02)$ & 5 & 0.02 \\
\hline 7 & RX J0511.7-0809 & 051141.55 & -080916.9 & I & $13.60(.02)$ & $0.96(.06)$ & $0.09(.05)$ & 3 & 0.15 \\
\hline 8 & 1RXS J051523.9-091234 & 051523.44 & -091246.1 & II & $11.67(.04)$ & $0.75(.01)$ & $0.27(.04)$ & 4 & 0.04 \\
\hline 9 & 1RXS J051631.5-080510 & 051631.32 & -080516.1 & II & $11.47(.03)$ & $0.67(.01)$ & $0.12(.02)$ & 4 & 0.06 \\
\hline 10 & RX J0517.0-0907 & 051700.73 & -090712.3 & II & $9.69(.03)$ & $0.64(.01)$ & $0.15(.01)$ & 4 & 0.0: \\
\hline 11 & RX J0517.9-0708 & 051754.40 & -070819.5 & II & $10.72(.01)$ & $0.85(.01)$ & $0.46(.02)$ & 4 & 0.04 \\
\hline 12 & RX J0523.1-0741 & 052302.62 & -074200.9 & III & $11.33(.01)$ & $0.67(.01)$ & $0.18(.02)$ & 5 & 0.0: \\
\hline 13 & 1RXS J052345.0-075343 & 052344.32 & -075337.4 & III & $12.25(.01)$ & $1.28(.01)$ & $1.19(.07)$ & 3 & 0.05 \\
\hline 14 & RX J0524.4-0640 & 052422.12 & -063915.9 & III & $12.27(.05)$ & $1.00(.01)$ & $0.67(.05)$ & 4 & 0.13 \\
\hline 15 & RX J0529.8-0102 & 052950.26 & -010208.9 & IV & $10.08(.02)$ & $0.20(.01)$ & $0.08(.01)$ & 9 & 0.0: \\
\hline 16 & RX J0530.1+0041 & 053005.18 & +004120.3 & - & $9.56(.03)$ & $0.65(.02)$ & - & 108 & - \\
\hline 17 & 1RXS J053114.3-092237 & 053114.17 & -092230.7 & VI & $10.48(.02)$ & $0.64(.01)$ & $0.07(.02)$ & 4 & 0.06 \\
\hline 18 & RX J0533.7-0521 & 053344.73 & -052103.8 & VI & $13.34(.09)$ & $0.81(.07)$ & $-0.41(.11)$ & 3 & - \\
\hline 19 & RX J0535.0-0618 & 053457.63 & -061831.4 & VI & $11.23(.04)$ & $0.66(.02)$ & $0.13(.03)$ & 6 & 0.06 \\
\hline 20 & RX J0536.1-0541 & 053606.33 & -054156.4 & VI & $11.68(.02)$ & $0.68(.02)$ & $0.05(.02)$ & 2 & 0.09 \\
\hline 21 & RX J0551.5-0628 & 055131.09 & -062908.5 & VII & $11.53(.01)$ & $0.55(.01)$ & $-0.01(.01)$ & 2 & - \\
\hline 22 & 1RXS J055841.6-031123 & 055841.66 & -031103.2 & VII & $11.76(.01)$ & $1.02(.01)$ & $0.71(.01)$ & 2 & 0.12 \\
\hline
\end{tabular}

${ }^{a}$ Coordinates are from USNO-B1 catalogue (Monet et al. 2003).

routine which finds, among our sample of standard stars, the spectrum which better matches the target spectrum.

Each standard spectrum, properly shifted in wavelength to the $R V$ of the target, has been progressively broadened by convolving it with rotational profiles of increasing $v \sin i$, using a step of $9 \mathrm{~km} \mathrm{~s}^{-1}$. At each step the rotationally-broadened and wavelength-shifted standard spectrum was subtracted to the observed one. The sum of the residuals, in the spectral range from 5860 to $6530 \AA$, has been taken as indicator for the goodness of the "fit". The standard star which gives the lowest sum of residuals provides the spectral type of the target. The spectral subtraction technique is illustrated in Figs. 3-7.

Some indication of the rotational velocity can be also gained with this technique. Indeed, given the intermediate resolution used, we can only distinguish between low and fast rotators and have a rough estimate of the rotational velocity, because the minimum $v \sin i$ which gives a detectable broadening of the template spectra is $\simeq 45 \mathrm{~km} \mathrm{~s}^{-1}$ (which corresponds to the pixel spacing of $0.9 \AA$ at the observed wavelengths).

We estimate an accuracy on the spectral type determination within 1-2 sub-classes. Stars with repeated observations yield consistent spectral types, if comparably good spectra are used. In the case of a double-lined spectroscopic binary, the spectrum synthesis method would yield inconsistent results, as different spectral types and different rotational velocities may be obtained at different orbital phases. This seems to be the case of RX J0500.7-0456, since, in addition to $R V$ variations, we also find very different $v \sin i$ on different dates (see Table 4).

For the latest-type star in our sample, RX J0502.0+0959, we were not able to obtain a reliable indication of its rotational velocity. However, the spectral type we deduce for this star $(\mathrm{M} 2 \mathrm{~V})$ is consistent with the $B-V$ color index.

\subsection{Sodium equivalent widths}

As a further diagnostic for spectral classification we have used the $\mathrm{NaID}_{2}$ lines, whose intensities change according to the temperature and gravity of the star. Since we have mediumresolution spectra, we could not analyse the individual $\mathrm{Na}_{\mathrm{ID}} \mathrm{D}_{2}$ line profiles, and we preferred to use their total equivalent width $\left(W_{\mathrm{D}_{2}}\right)$ integrating over the whole sodium doublet. The results are displayed in Fig. 8, where the $\mathrm{NaID}_{2}$ equivalent widths for main-sequence, sub-giant, and giant standard stars are plotted versus the $B-V$ color using different symbols. The $\mathrm{NaID}_{2}$ equivalent widths follow different trends with the $B-V$ color (i.e. as a function of effective temperature) for stars of different luminosity class. In the same figure the target stars are also plotted with plus symbols. Nearly all the late-type targets $(B-V>0.7)$ fall close to the locus of main sequence stars (solid line in Fig. 8), with the exception of 1RXS J051523.9-091234 and RX J0524.4-0640 that are located close to the giant star sequence (dashed line).

\subsection{H $\alpha$ equivalent widths}

The $\mathrm{H} \alpha$ equivalent widths (EW) were obtained by integration of the line profile on the normalized spectra. This integration was performed by linear interpolation of the continuum across the line. This method was used for both emission and absorption-line spectra and the EW values $\left(W_{\mathrm{H} \alpha}\right)$ have been 
Table 4. Spectral types, radial velocities, $\mathrm{H} \alpha$ and Li I equivalent widths of PMS candidates.

\begin{tabular}{|c|c|c|c|c|c|c|c|c|c|}
\hline 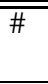 & "RX/1RXS & Sp.T. & $\begin{array}{r}v \sin i \\
\left(\mathrm{~km} \mathrm{~s}^{-1}\right) \\
\end{array}$ & $\begin{array}{c}\text { HJD } \\
(2450000+)\end{array}$ & $\begin{array}{c}R V \\
\left(\mathrm{~km} \mathrm{~s}^{-1}\right)\end{array}$ & $\begin{array}{l}W_{\mathrm{D}_{2}} \\
(\AA)\end{array}$ & $\begin{array}{l}W_{\mathrm{H} \alpha} \\
(\AA)\end{array}$ & $\begin{array}{l}W_{\mathrm{H} \alpha}^{\mathrm{em}} \\
(\AA)\end{array}$ & $\begin{array}{l}W_{\mathrm{LiI}} \\
(\AA)\end{array}$ \\
\hline \multirow[t]{3}{*}{1} & J0500.7-0456 & F9V & 61 & 1885.4977 & $12.0 \pm 17.9$ & 1.28 & 4.85 & $0.05 \pm 0.03$ & - \\
\hline & & & 139 & 1889.5684 & $-14.5 \pm 18.2$ & 1.39 & 4.45 & $0.14 \pm 0.15$ & - \\
\hline & & & 157 & 1889.5937 & $-20.8 \pm 16.8$ & 1.16 & 4.41 & $0.10 \pm 0.13$ & - \\
\hline \multirow[t]{2}{*}{2} & $\mathrm{~J} 0502.0+0959$ & $\mathrm{M} 2 \mathrm{~V}$ & - & 1891.4770 & $31.0 \pm 100.7$ & 9.62 & -6.95 & $7.51 \pm 0.45$ & - \\
\hline & & & & 1896.4947 & $59.4 \pm 244.6$ & 9.88 & -7.88 & $8.20 \pm 0.53$ & - \\
\hline 3 & J0502.4-0744 & G2V & $<45$ & 1894.4014 & $20.2 \pm 5.8$ & 1.42 & 1.93 & $0.59 \pm 0.08$ & $0.26 \pm 0.08$ \\
\hline \multirow[t]{3}{*}{4} & $\mathrm{~J} 0503.1+0548$ & $\mathrm{G} 2 \mathrm{~V}$ & 70 & 1891.5131 & $-59.8 \pm 7.7$ & 1.81 & 1.71 & $1.18 \pm 0.14$ & - \\
\hline & & & 78 & 1893.4661 & $-35.8 \pm 12.5$ & 1.50 & 1.68 & $0.92 \pm 0.14$ & - \\
\hline & & & 96 & 1896.6076 & $-64.8 \pm 15.6$ & - & 1.08 & $1.48 \pm 0.41$ & - \\
\hline \multirow[t]{3}{*}{5} & $\mathrm{~J} 050315.2+084101$ & K5V & $<45$ & 1891.4377 & $17.0 \pm 8.1$ & 6.75 & 0.27 & $0.67 \pm 0.11$ & - \\
\hline & & & & 1895.4057 & $1.5 \pm 19.3$ & 6.99 & 0.30 & $0.84 \pm 0.35$ & - \\
\hline & & & & 1896.5330 & $14.9 \pm 8.7$ & - & 0.23 & $0.78 \pm 0.21$ & - \\
\hline \multirow[t]{2}{*}{6} & J0510.7-0316 & F9V & 52 & 1891.5533 & $8.8 \pm 13.6$ & 1.27 & 2.62 & $0.92 \pm 0.38$ & - \\
\hline & & & & 1891.5869 & $20.3 \pm 13.1$ & 1.04 & 2.78 & $0.61 \pm 0.25$ & - \\
\hline \multirow[t]{2}{*}{7} & J0511.7-0809 & $\mathrm{K} 2-3 \mathrm{~V}$ & $<45$ & 1897.4058 & $32.0 \pm 16.9$ & 6.36 & -2.06 & $3.10 \pm 0.68$ & $0.12: \pm 0.11:$ \\
\hline & & & & 1897.4409 & $16.9 \pm 13.6$ & 5.77 & -2.79 & $4.07 \pm 0.74$ & $0.44: \pm 0.17:$ \\
\hline 8 & J051523.9-091234 & G2V & $<45$ & 1894.4468 & $9.5 \pm 9.8$ & 1.34 & 2.03 & $0.89 \pm 0.17$ & $0.14 \pm 0.08$ \\
\hline 9 & J051631.5-080510 & G3V & 52 & 1892.4519 & $8.8 \pm 10.5$ & 1.83 & 2.32 & $1.00 \pm 0.28$ & $0.17 \pm 0.11$ \\
\hline \multirow[t]{2}{*}{10} & J0517.0-0907 & $\mathrm{G} 2 \mathrm{~V}$ & $<45$ & 1889.5064 & $24.6 \pm 5.5$ & 1.60 & 2.81 & $0.20 \pm 0.08$ & $0.16 \pm 0.05$ \\
\hline & & & & 1889.5309 & $19.9 \pm 7.4$ & 1.58 & 2.93 & $0.16 \pm 0.05$ & $0.19 \pm 0.04$ \\
\hline \multirow[t]{4}{*}{11} & J0517.9-0708 & $\mathrm{K} 0 \mathrm{~V}$ & $<45$ & 1890.5269 & $18.4 \pm 11.6$ & 3.45 & 1.31 & $0.64 \pm 0.12$ & $0.27 \pm 0.10$ \\
\hline & & & & 1894.6124 & $9.5 \pm 6.4$ & 3.23 & 1.24 & $0.78 \pm 0.21$ & $0.20 \pm 0.06$ \\
\hline & & & & 1895.6309 & $10.8 \pm 13.7$ & - & 1.16 & $0.97 \pm 0.21$ & $0.12 \pm 0.10$ \\
\hline & & & & 1896.4074 & $16.9 \pm 5.9$ & 3.34 & 1.11 & $0.82 \pm 0.13$ & $0.27 \pm 0.06$ \\
\hline 12 & J0523.1-0741 & F9V & $<45$ & 1892.4946 & $13.5 \pm 9.2$ & 1.34 & 1.79 & $1.32 \pm 0.29$ & $0.17 \pm 0.09$ \\
\hline \multirow[t]{3}{*}{13} & J052345.0-075343 & K5V & $<45$ & 1892.3822 & $21.4 \pm 19.9$ & 8.55 & -0.73 & $1.58 \pm 0.23$ & - \\
\hline & & & & 1892.4169 & $26.1 \pm 17.8$ & 8.58 & -0.82 & $1.85 \pm 0.26$ & - \\
\hline & & & & 1897.5182 & $31.7 \pm 18.4$ & 8.33 & -0.91 & $1.97 \pm 0.21$ & $0.19 \pm 0.07$ \\
\hline 14 & J0524.4-0640 & G5III-IV & 52 & 1893.5095 & $26.3 \pm 9.2$ & 2.57 & -0.19 & $1.69 \pm 0.45$ & $0.41 \pm 0.19$ \\
\hline 15 & J0529.8-0102 & A7 & - & 1885.5286 & $17.3 \pm 69.7$ & 1.25 & 10.75 & - & - \\
\hline 16 & $\mathrm{~J} 0530.1+0041$ & G3V & 52 & 1893.5438 & $43.1 \pm 10.4$ & 1.97 & -2.40 & $0.96 \pm 0.15$ & $0.28 \pm 0.06$ \\
\hline \multirow[t]{2}{*}{17} & J053114.3-092237 & F9V & $<45$ & 1890.5834 & $11.9 \pm 11.7$ & 1.35 & 2.16 & $0.98 \pm 0.18$ & $0.15 \pm 0.09$ \\
\hline & & & & 1890.6138 & $14.6 \pm 13.9$ & 1.33 & 2.00 & $1.08 \pm 0.19$ & $0.15 \pm 0.10$ \\
\hline 18 & J0533.7-0521 & F9V: & $<45$ & 1896.4499 & $31.5 \pm 29.4$ & -3.17 & -26.33 & $25.45 \pm 0.81$ & - \\
\hline \multirow[t]{3}{*}{19} & J0535.0-0618 & G2V & 61 & 1892.5301 & $36.2 \pm 10.6$ & 1.41 & 1.15 & $1.93 \pm 0.34$ & $0.45 \pm 0.15$ \\
\hline & & & & 1892.5612 & $22.3 \pm 8.4$ & 1.36 & 0.61 & $3.09 \pm 0.27$ & $0.20 \pm 0.11$ \\
\hline & & & & 1895.5499 & $36.9 \pm 12.0$ & 1.10 & 1.35 & $1.72 \pm 0.24$ & $0.22 \pm 0.08$ \\
\hline \multirow[t]{2}{*}{20} & J0536.1-0541 & G3V & 52 & 1893.4238 & $32.8 \pm 10.6$ & 1.33 & -0.85 & $2.69 \pm 0.29$ & $0.39 \pm 0.12$ \\
\hline & & & & 1896.5710 & $31.2 \pm 25.9$ & - & -3.41 & $5.14 \pm 0.30$ & $0.37 \pm 0.34$ \\
\hline \multirow[t]{2}{*}{21} & J0551.5-0628 & F8-9V & 87 & 1894.5735 & $20.0 \pm 18.6$ & 0.91 & 1.84 & $0.93 \pm 0.22$ & - \\
\hline & & & 52 & 1897.5919 & $17.5 \pm 12.9$ & 1.02 & 2.77 & $0.69 \pm 0.12$ & - \\
\hline \multirow[t]{2}{*}{22} & J055841.6-031123 & K3-5 V & $<45$ & 1894.4932 & $-10.3 \pm 8.4$ & 5.70 & -1.19 & $2.18 \pm 0.22$ & - \\
\hline & & & & 1897.4805 & $-72.5 \pm 12.2$ & 5.60 & -0.93 & $1.74 \pm 0.25$ & - \\
\hline
\end{tabular}

1 Suspected spectroscopic binary, based on $R V$ variations and variable CCF peak width. No lithium is observed.

2 Very active dMe star on the field of Orion SFR, but much closer to us as deduced from the Hipparcos distance of $32 \mathrm{pc}$.

3 PMS star already studied by Alcalà et al. $(1996,2000)$ who assigned spectral type $\mathrm{G} 6, v \sin i=26 \mathrm{~km} \mathrm{~s}^{-1}$, and $W_{\mathrm{Li}}=305 \mathrm{~m} \AA$.

4 No lithium absorption is detected, and the $R V$ measurements are not consistent with the SFR but suggest a possible spectroscopic binary.

7 At about 1'.5 from IRAS 05092-0814. Lithium EW just on the Pleiades upper envelope. $R V$ might be consistent with the Orion SFR.

9 Lithium just over the Pleiades upper envelope. $R V$ and kinematics consistent with Orion SFR.

$10 R V$ consistent with the SFR, but very high proper motion. Lithium just on the Pleiades border.

11 From high resolution spectroscopy, Alcalà et al. (2000) derived spectral class $K 2$, and $W_{\mathrm{i}} \simeq 280 \mathrm{~m} \AA$.

13 Possible post-T Tauri. $R V$ consistent with the SFR.

14 Likely PMS star. $R V$ consistent with the SFR. Both spectral appearance and sodium EW indicate a gravity sensibly lower than for MS stars of the same color, suggesting a very young age.

15 Early-type star with $R V$ consistent with the SFR. A photometric parallax of about $330 \mathrm{pc}$ is found if the star is assumed to lie on the ZAMS.

17 Lithium just on the Pleiades border, while kinematics is consistent with the Orion SFR.

16 Already observed by Alcalà et al. $(1996,2000)$, who report spectral type $\mathrm{G} 2$ and $W_{\mathrm{Li}}=260 \mathrm{~m} \AA$.

18 V730 Ori. Faint star embedded into a dense nebular region. Strong emission in $\mathrm{H} \alpha$ and forbidden lines possibly arise from the surrounding medium. No lithium absorption is seen.

19 Strong lithium line. $R V$ and space location fairly consistent with the Orion SFR.

20 Parenago 2328. Embedded into a nebular region. High lithium content.

$22 R V$ variable. $\mathrm{H} \alpha$ in emission, but no lithium. 


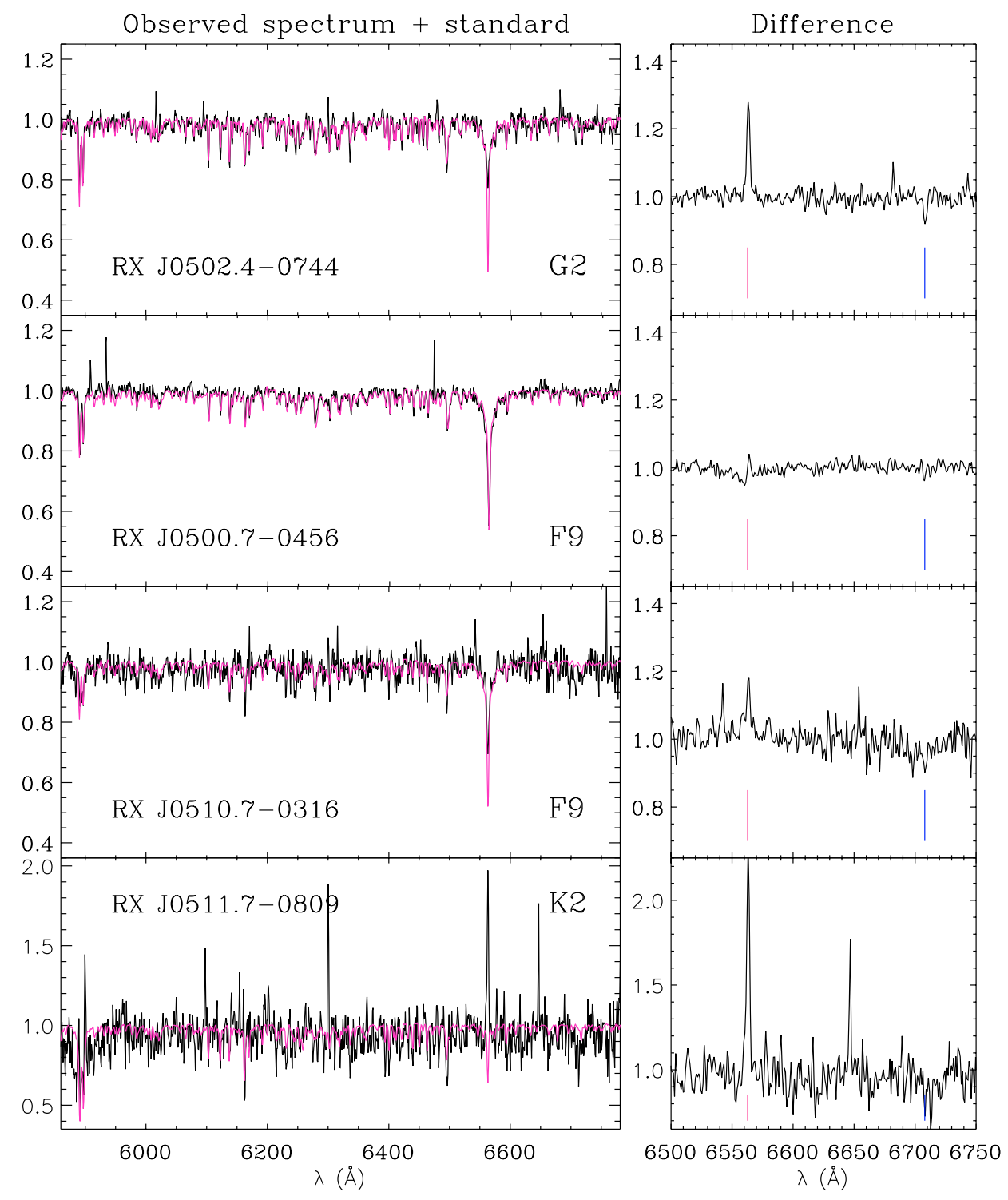

Fig. 3. Left panels. Observed spectra of four stars in our sample (thick lines) with the standard star spectra which give the best match superimposed (thin dotted lines). Right panels. Difference between observed and standard-star spectra in the H $\alpha$ region. The wavelengths of $\mathrm{H} \alpha$ and Li I lines are also marked in the same panels.

reported in Table 4, where negative numbers indicate emission lines.

In addition to the total $\mathrm{H} \alpha \mathrm{EW}$, we measured the "net" $\mathrm{H} \alpha \mathrm{EW}$ on the residual spectra, after the subtraction of the spectral-type template. In this case the photospheric absorption lines cancel out to a large extent, with the only remaining features being the $\mathrm{H} \alpha$ emission core (see e.g. Frasca et al. 1994, 2000) and, eventually, the LiI absorption line. We estimated the error on the net $\mathrm{H} \alpha \mathrm{EW}$ as the product of the continuum placement error (evaluated from the rms of the difference spectrum in two windows at the two sides of $\mathrm{H} \alpha$ ) with the integration range. The net $\mathrm{H} \alpha$ equivalent width, $W_{\mathrm{H} \alpha}^{\mathrm{em}}$, listed in Table 4 is always a non-negative number (unless excess-absorption is observed). All the optical counterparts of the studied RASS sources show the $\mathrm{H} \alpha$ line as a pure emission feature or filled in with emission in the core. This stellar activity signature guarantees the correct identification of the optical counterparts. In addition, all the solar-type targets, with the exception of RX J0517.0-0907, display $W_{\mathrm{H} \alpha}^{\mathrm{em}}$ values greater than or close to those measured for the already confirmed PMS stars RX J0502.4-0744 and RX J0530.1+0041. The average value found for RX J0517.0-0907, $W_{\mathrm{H} \alpha}^{\mathrm{em}}=0.18 \AA$, is close to that found for HD $206860\left(W_{\mathrm{H} \alpha}^{\mathrm{em}}=0.11-0.16 \AA\right)$ a young ( $\approx 300$ Myr) G0 main sequence star (Frasca et al. 2000).

Notwithstanding the moderate resolution, the spectra of several targets display, in the "observed-standard" residual, a broad $\mathrm{H} \alpha$ emission. In at least two cases, RX J0523.1-0741 and RX J0536.1-0541, an asymmetric blue $\mathrm{H} \alpha$ wing has been also observed. The full widths of such profiles, converted into velocity scale, are of the order of $100-200 \mathrm{~km} \mathrm{~s}^{-1}$.

Broad asymmetric $\mathrm{H} \alpha$ profiles are frequently observed in late-type PMS stars and are commonly interpreted as due to 


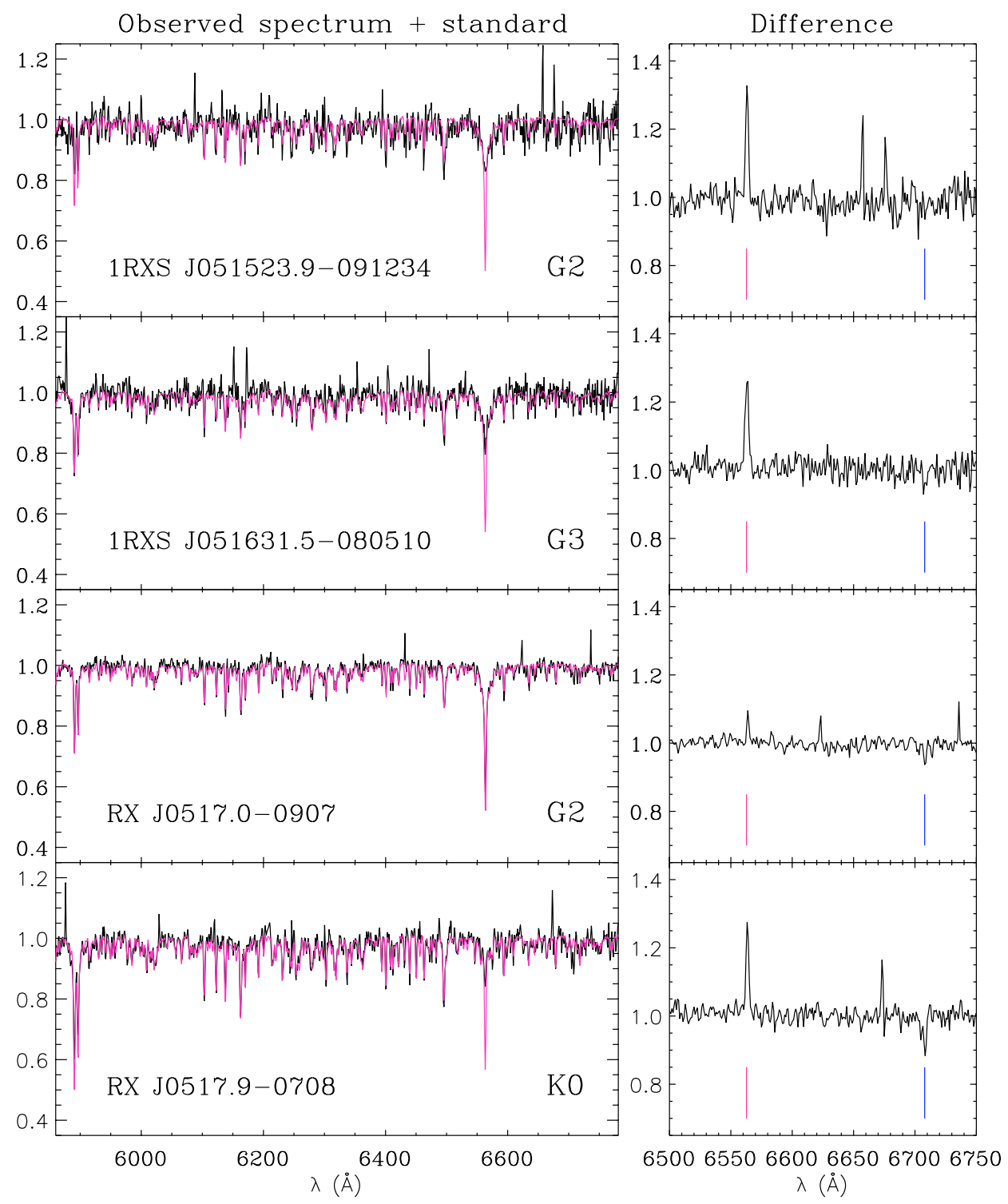

Fig. 4. Same as in Fig. 3.

accretion from the circumstellar disk superimposed on the chromospheric emission (e.g. Bailey 1998; Takami et al. 2003). Takami et al. (2003), for example, report on blue-shifted outflows in the range $-100 \div-300 \mathrm{~km} \mathrm{~s}^{-1}$ and up to $-700 \mathrm{~km} \mathrm{~s}^{-1}$ for Z CMa.

The observation of broad and asymmetric $\mathrm{H} \alpha$ wings in several of our targets gives strong support to their PMS nature.

\subsection{Lithium equivalent widths}

The same procedure used for the measurement of $W_{\mathrm{H} \alpha}^{\mathrm{em}}$ has been applied to the measurement of the Li I ( $\lambda 6708 \AA)$ line equivalent width $\left(W_{\mathrm{LiI}}\right)$. The advantage of the subtraction of the spectral-type template in this case is to remove from the observed spectrum absorption lines, that are blended with the Li I line at this spectral resolution.

We could measure the Li I EW only on the best exposed spectra. The errors in the Li I EW were evaluated as above. These values are also listed in Table 4.
The Li I EW versus $(B-V)_{0}$ diagram for the stars in our sample is shown in Fig. 9 (filled circles). Li I EW values for Pleiades stars (Soderblom 1993) are also marked with plus signs and their upper envelope is displayed by a solid line. We have used this observational boundary to select the very young PMS stars in our sample against ZeroAge Main Sequence (ZAMS) ones, with an age of a few hundreds Myr, that are contaminating the Orion sample and are probably associated to the Gould Belt. This criterion has been adopted by several authors to distinguish between PMS and young MS stars (cf. Martín \& Magazzù 1999; Preibisch et al. 1998; Covino et al. 1997).

Of the 13 stars in our sample, observed with sufficient S/N to detect and measure the $\mathrm{Li}$ I- $\lambda 6708$ line, 6 have Li I EW well above the Pleiades upper boundary and are bona-fide PM stars. They are represented in Fig. 9 with big symbols. Of these six stars, however, only 4 can be considered as new WTTS, whereas two, namely RX J0502.4-0744 and RX J0530.1+0041, were previously known WTTS and were reobserved by us and 


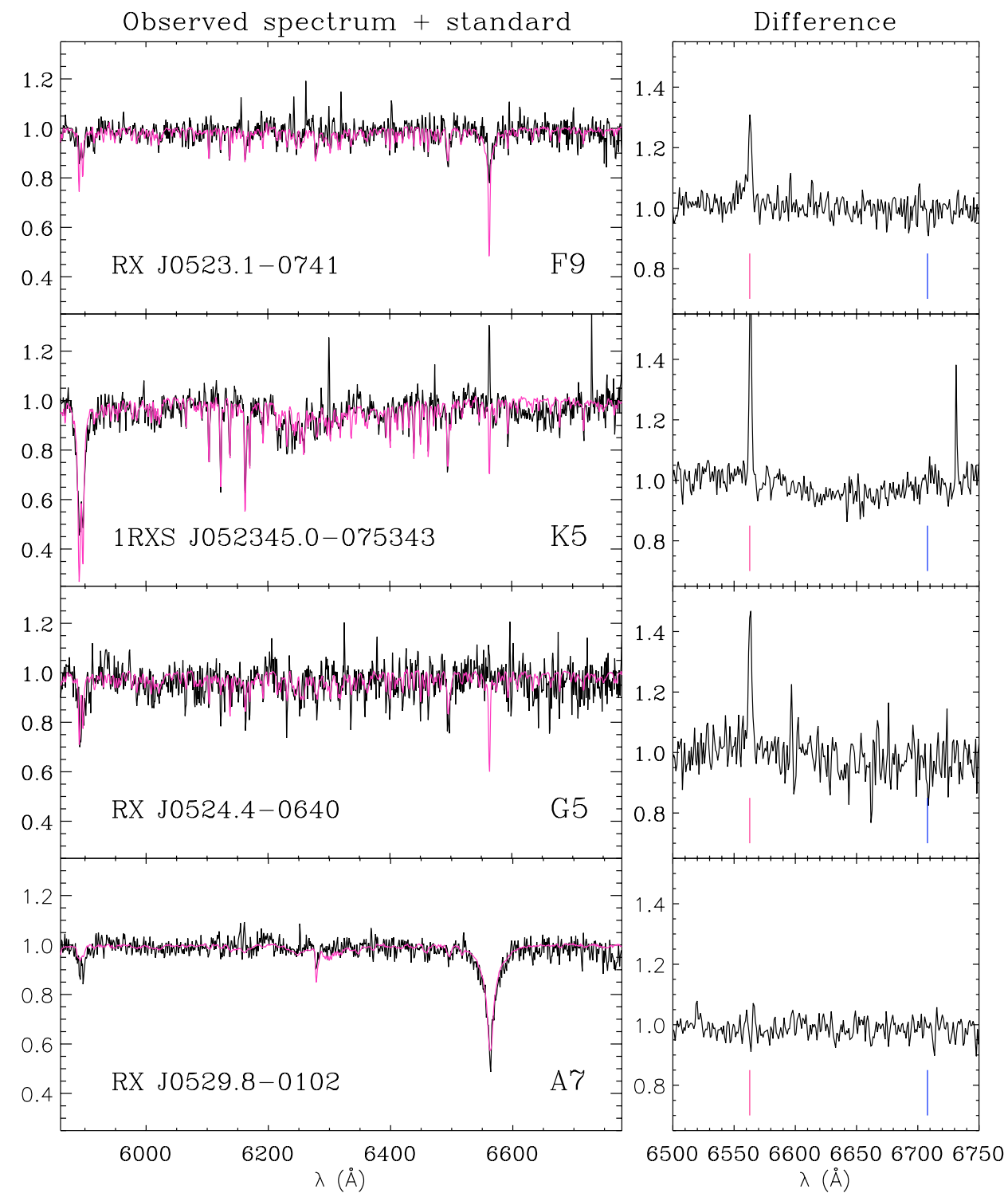

Fig. 5. Same as in Fig. 3.

used here to test our method. The other seven stars with detectable Li I $\lambda 6708$ line fall below or near to the Pleiades upper envelope and are marked with smaller circles. However, five of these stars have $W_{\mathrm{LiI}}$ very close to the upper envelope in Fig. 9, thus their PMS nature can be neither assessed nor excluded on the basis of the lithium test only and additional criteria, e.g. kinematics, should be taken into account.

It is interesting to note that 1RXS J052345.0-075343 falls in the PTTS region defined by Martín (1997). However, to confirm this finding, its lithium content should be verified by means of higher resolution spectroscopy.

\section{Spatial distribution and kinematics}

The first extensive study of proper motions of stars in the Orion region, covering a sky area of $3 \times 3$ square degrees, was made by Parenago (1954). McNamara \& Huels (1983) further analysed the Parenago's proper motions to derive membership probabilities. More recent studies of proper motions have been presented by Jones \& Walker (1988), van Altena et al. (1988), McNamara et al. (1989), and Tian et al. (1996). In all these works, relatively small sky regions, covering areas less than 7 square degrees around the Orion Nebula Cluster (ONC), were selected. Proper motion accuracy as small as $0.3 \mathrm{mas} \mathrm{yr}^{-1}$ has been achieved. All the cited authors found consistent values of the mean cluster proper motion and very close to $\mu_{x}=0.0 \mathrm{mas} \mathrm{yr}^{-1}, \mu_{y}=0.0 \mathrm{mas} \mathrm{yr}^{-1}$. They also gave membership probability by analysing the peaks in the proper motion distribution.

Proper motion studies of stars in a wider area around the Orion complex based on Hipparcos data have been devoted to the Ori OB1 association (e.g. de Zeeuw et al. 1999). Ori OB1 is one of the best studied nearby OB association but, unfortunately, it is located close to the Solar Antapex, and its space motion is almost purely radial with respect to the Sun. The proper motions of the stars members of Orion OB1 association are consequently small and rather randomly distributed. De Zeeuw et al. (1999) show that the proper motion 


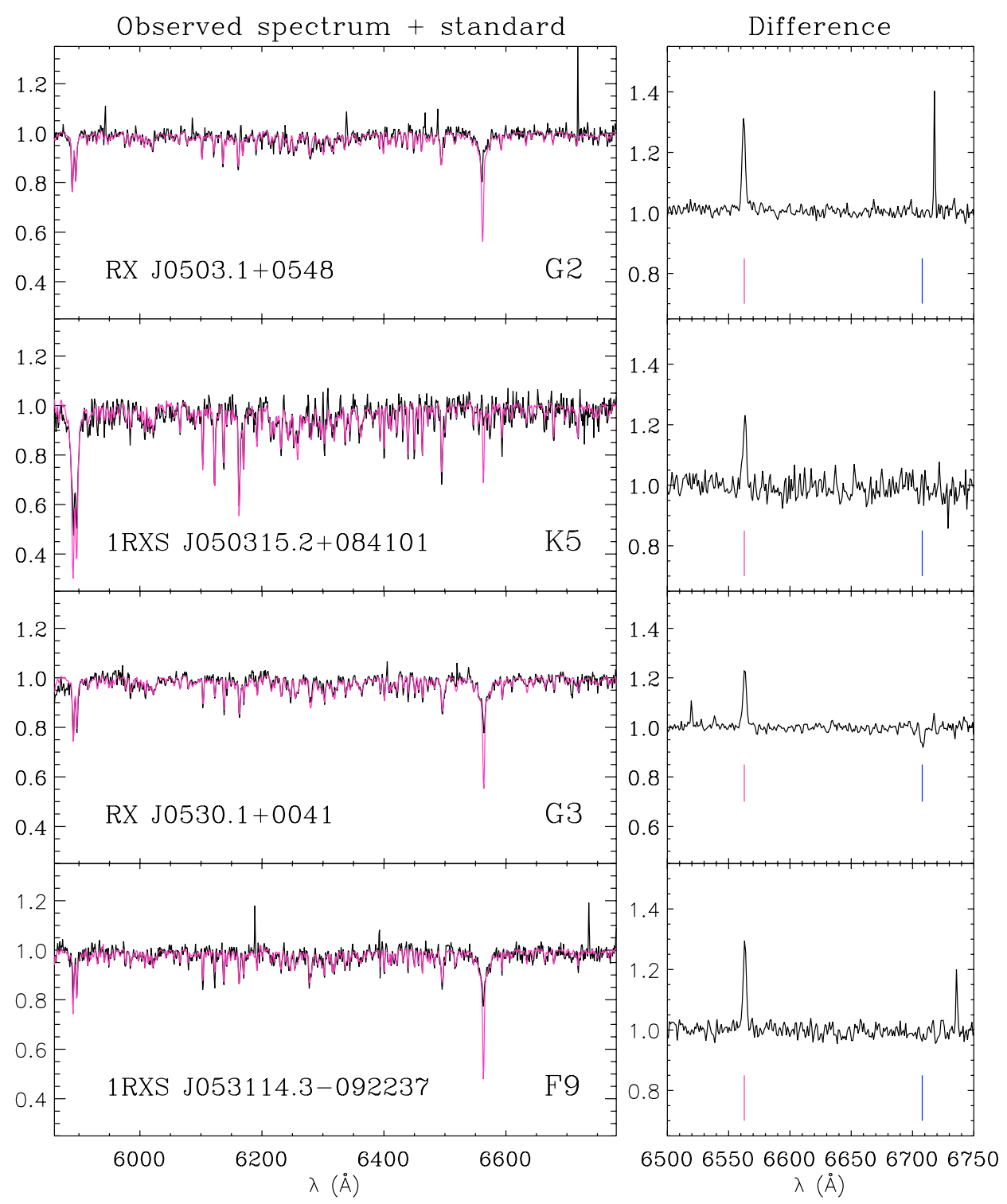

Fig. 6. Same as in Fig. 3.

distribution of the members of Ori OB1 association is centered

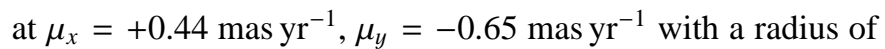
about $5 \mathrm{mas} \mathrm{yr}^{-1}$. The central value of $\mu_{x}$ and $\mu_{y}$ are consistent with those found for the ONC members, but the dispersion appears larger.

With the aim to investigate the kinematical link between active stars in the general direction of Orion and those associated to the ONC, as well as to have additional tools for the selection of PMS stars, we examined the kinematical properties of our RASS sample and of the stars selected by Alcalá et al. (2000), which are distributed over a wide area around the ONC.

Unfortunately, all our targets, with the only exception of RX J0502.0+0959, are not included into the Hipparcos main catalogue (ESA 1997), but some of them have been observed by Tycho. Although the Tycho parallaxes have very large errors and cannot be taken into account, the proper motions can be used.

We found in the Tycho-2 catalogue (Hog et al. 2000) the proper motions of 13 stars in our sample (including the two previously known PMS stars) and of 50 PMS stars among those selected by Alcalá et al. (2000) in the Orion region. The typical precision on proper motion measurements of Tycho- 2 entries is

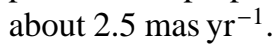

In Fig. 10 the space distribution of the stars in the Alcalá et al. sample (asterisks) and in the present sample (big dots for lithium-rich stars, small dots for stars with a "Pleiades-like" lithium content, and open circles for stars with no lithium detected) is reported over the $100 \mu \mathrm{m}$ IRAS map displayed as a grey level image. The arrows indicate the proper motions. In the figure the Orion 1a-b-c clusters, the $\lambda$ Ori region and the new $\sigma$ Ori cluster (Blaauw 1991) are also indicated by dashed contours.

Three stars, at around right ascention $\alpha=5^{\mathrm{h}} .17$ and declination $\delta=-3.5$, have consistent proper motions and coincide in position with the reflection nebula NGC 1788, a region harboring many new low-mass PM stars (Alcalá et al. in preparation). Other four stars at $\alpha \approx 5.65$ and $\delta \approx+9.5$ also have coherent proper motions and their position approximately coincide with 


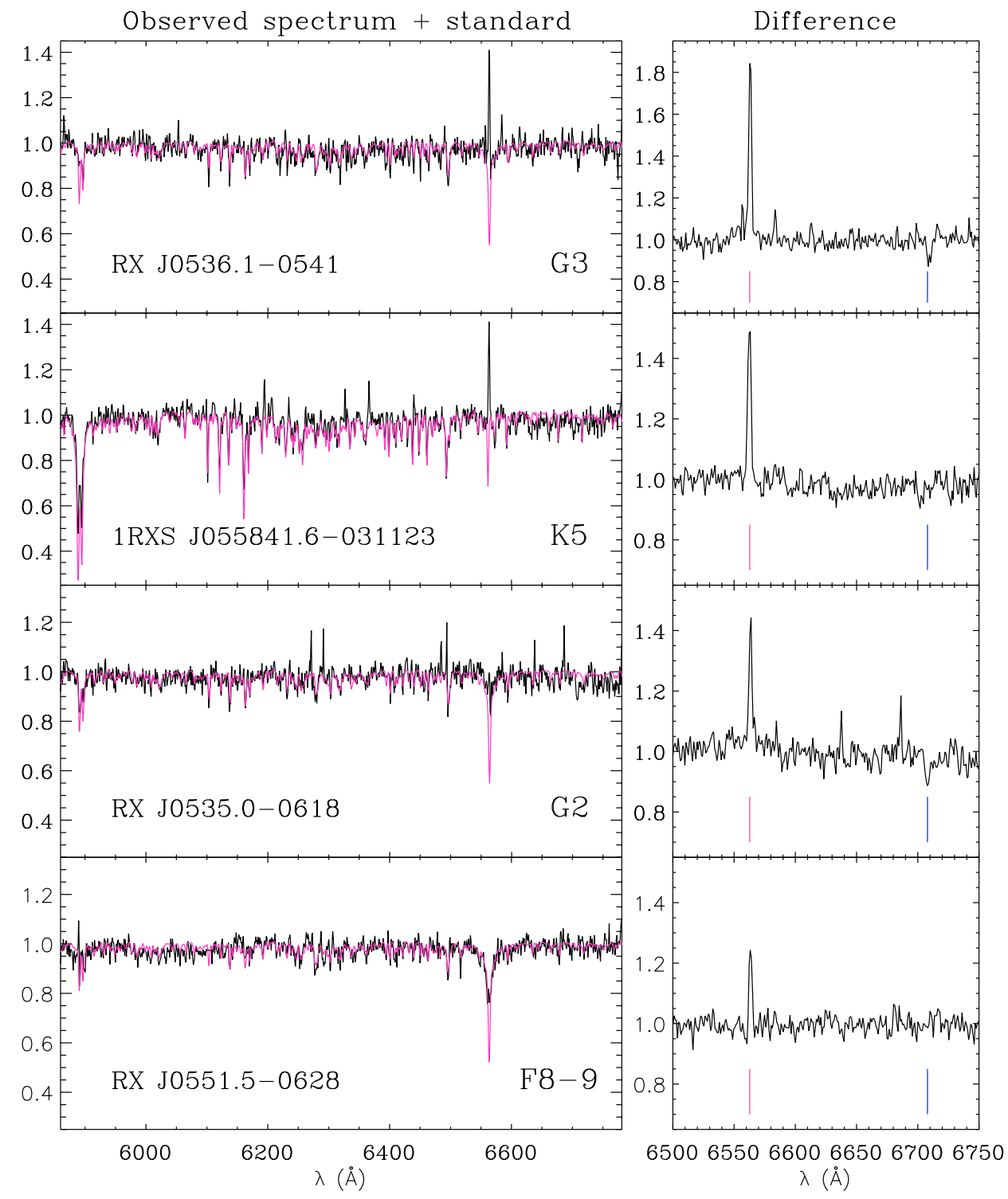

Fig. 7. Same as in Fig. 3.

a $\mathrm{CO}$ enhancement, and with the regions 4 and 5 studied by Dolan \& Mathieu (2001), in the $\lambda$ Orionis region. Also, some lithium-rich stars with coherent proper motions, at around right ascention $\alpha=5.3$, and declination $\delta=-8^{\circ}$, i.e. south-west of the ONC, can be noticed, in the same area where some faint $\mathrm{CO}$ condensations are present, although they are slightly more scattered. These regions, as well as many others around the Orion complex, were found to coincide with overdensities of X-ray sources having a high probability of being lowmass PMS stars (e.g. Sterzik et al. 1995; Walter et al. 2000). In priciple, this could be considered as an evidence supporting the hypothesis that the distributed T Tauri stars in SFRs are formed in cloudlets, as the Feigelson's (1996) simulations predict groups of PMS stars with coherent proper motions. However, the statistics on the PMS population of these small regions is still rather poor and hence, it is not clear whether these "clumps" correspond to the predicted cloudlet regions.

The proper motions diagram, displayed in Fig. 11, shows a roughly circular distribution whose "central" coordinates

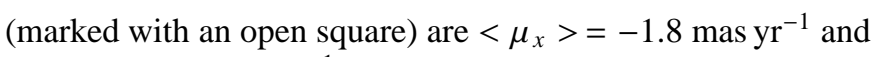

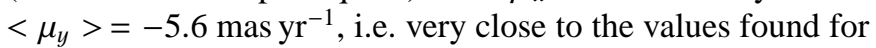
the ONC and Ori OB1 association, within the errors of Tycho proper motions. We determined these values from the distributions of $\mu_{x}$ and $\mu_{y}$ for all stars with detectable lithium by fitting gaussian functions whose sigmas are 6.4 and 6.9 mas yr$^{-1}$, respectively.

The big circle in Fig. 11 defines the outer limit given by Eq. (8) of de Zeeuw et al. (1999) for membership to the Ori OB1 association.

Notwithstanding the limited sample (54 objects) and the errors of Tycho-2 proper motions, considerably greater than those typically measured for ONC stars, which prevent us from doing a detailed analysis and discussion, we point out that the proper-motion distribution of the stars in our sample shows a dispersion significantly larger than that found for ONC members and comparable with that for hot stars of the Ori OB1 association. 


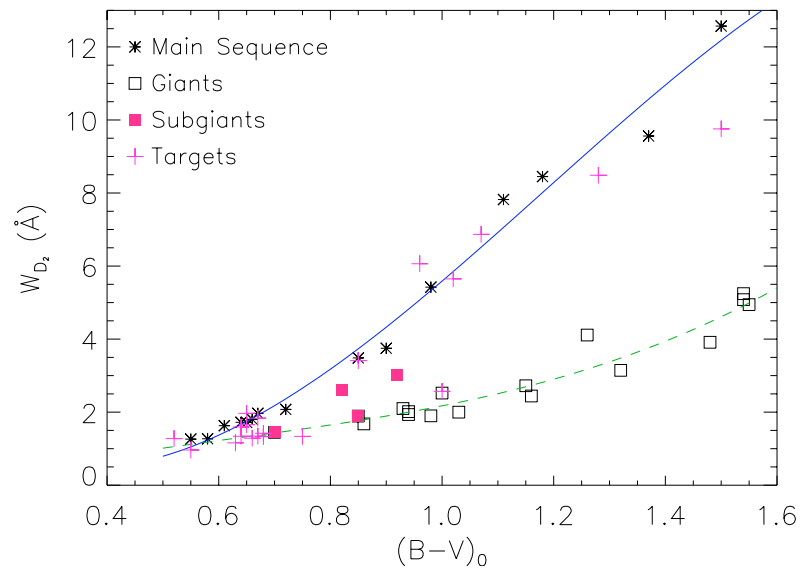

Fig. 8. Sodium $\mathrm{D}_{2}$ lines equivalent width plotted as a function of $B-V$ color index for the standard stars (asterisks and squares for mainsequence and evolved stars, respectively) and PMS candidates (plus symbols). Polynomial fits to main sequence and giant stars are also displayed by continuous and dashed lines, respectively.

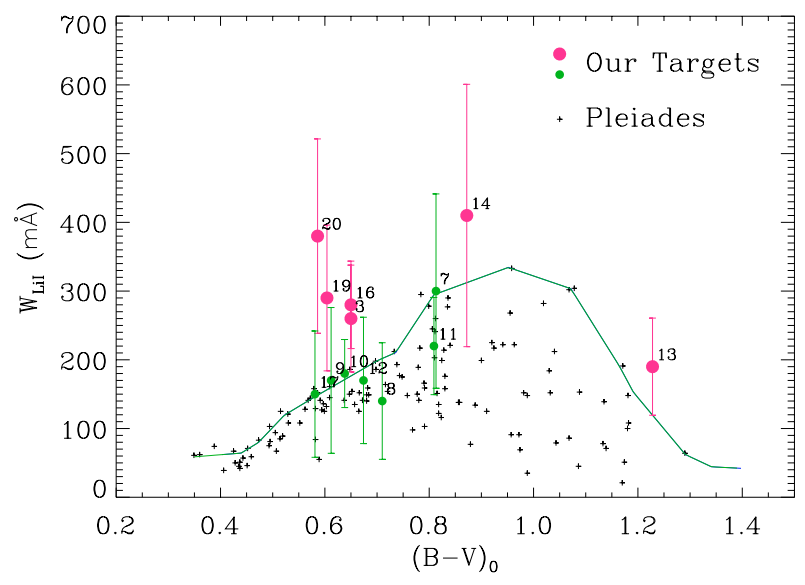

Fig. 9. Li I equivalent width of our PMS candidates plotted as a function of $(B-V)_{0}$ color index (filled circles). Li I EWs for Pleiades stars (Soderblom et al. 1993) are represented by plus symbols. The upper envelope of these latter EWs is marked with a full line. The points are labelled according to the code given in Tables 1, 3 and 4.

This supports the hypothesis that many of the stars in our sample could indeed be related with the Orion SFR, sharing with ONC members the same average proper motion, although with a larger dispersion, which might indicate a mixture of young stars, still associated, kinematically and spatially, to the gas clouds that generated them, with relatively older ones, probably belonging to the Gould belt population. The radial velocities provide additional support to this hypothesis.

A spectroscopic study of 61 late-type stars in the Orion Nebula was presented by Walker (1983). The mean radial velocity of this sample of stars is $+32 \mathrm{~km} \mathrm{~s}^{-1}$, and is independent of spectral type. Walker found a radial velocity dispersion of about $24 \mathrm{~km} \mathrm{~s}^{-1}$ for the stars identified as cluster members, with some indication of increase of the dispersion in the radial velocity distribution for later spectral types.

The $R V$ distribution of the RASS stars in Orion studied by Alcalá et al. (2000) shows a double peak suggesting two kinematical groups. The two peaks are at +18.5 and $+25 \mathrm{~km} \mathrm{~s}^{-1}$ with velocity dispersion (1 sigma) of 3.0 and $4.5 \mathrm{~km} \mathrm{~s}^{-1}$, respectively. The RASS stars with $R V>21 \mathrm{~km} \mathrm{~s}^{-1}$ tend to be more correlated, both spatially and kinematically, with the regions of stronger $\mathrm{CO}$ emission of the Orion $\mathrm{A}$ and $\mathrm{B}$ clouds (Maddalena et al. 1986) and with the Ori OB1a association, while the others appear more dispersed and are believed to belong to the sparse star population of the Gould Belt.

The errors on our $R V$ measurements, typically about $10 \mathrm{~km} \mathrm{~s}^{-1}$, prevent us to make such a fine discrimination. However, with the exception of four stars, all the targets have RV compatible with that of the Orion SFR. We remark that 3 out of these four exceptions, namely RX J0500.7-0456, RX J0503.1+0548, and 1RXS J055841.6-031123 are probable spectroscopic binaries, while the other, RX J0502.0+0959, is a field M star apparently unrelated to the SFR, as witnessed by its Hipparcos parallax. Then, the radial velocities of our sample do not allow us to exclude any candidate as a possible member of the Orion SFR.

Unfortunately, among the lithium-rich stars in our sample, only the previously known WTTS RX J0530.1+0041 and RX J0502.4+0744 have Tycho proper motions, but the remaining four stars have $R V \mathrm{~s}$ very well consistent with the SFR. In addition, four out of five stars with lithium equivalent width close to the Pleiades upper envelope, namely RX J0511.7-0809, 1RXS J051631.5-080510, RX J0523.10741, and 1RXS J053114.3-092237, have all $R V$ s and proper motions well consistent with the SFR, and they deserve to be observed spectroscopically at higher resolution in order to obtain a more precise determination of their lithium content. The proper motion of the star RX J0517.0-0907, instead, falls too far away from the cluster of points in Fig. 11.

\section{HR diagram}

We have examined the evolutionary status of the stars in our sample with detectable lithium, by their position in the HR diagram based on our $U B V$ photometry and spectral type determination. Since no precise parallaxes are available for these stars, with the only exception of RX J0502.0+0959, we have adopted for all of them the distance of $460 \mathrm{pc}$ for the Orion complex (Genzel \& Stutski 1989). For RX J0502.0+0959 we adopted the Hipparcos distance of $32 \mathrm{pc}$.

For the determination of the effective temperatures, the $T_{\text {eff }}$ versus $B-V$ calibration of Flower (1996) was used. The bolometric correction, tabulated as a function of $T_{\text {eff }}$ in the same work, was used to convert the $V$ magnitudes to bolometric ones. The luminosities of the PMS candidates were calculated assuming a bolometric magnitude of 4.74 for the Sun (Cox 2000) and the distance of the Orion complex (460 pc).

Figure 12 shows the position on the HR diagram of the stars in our sample with different symbols, according to their lithium content. The theoretical PMS evolutionary tracks by D'Antona and Mazzitelli (1997) (MLT + Alexander's opacities set) are also overplotted.

Assuming that these objects are at the Orion distance, they are younger than $10 \mathrm{Myrs}$ and their masses are in the range from 0.8 to $2 M_{\odot}$. Note that the position of the dMe star 


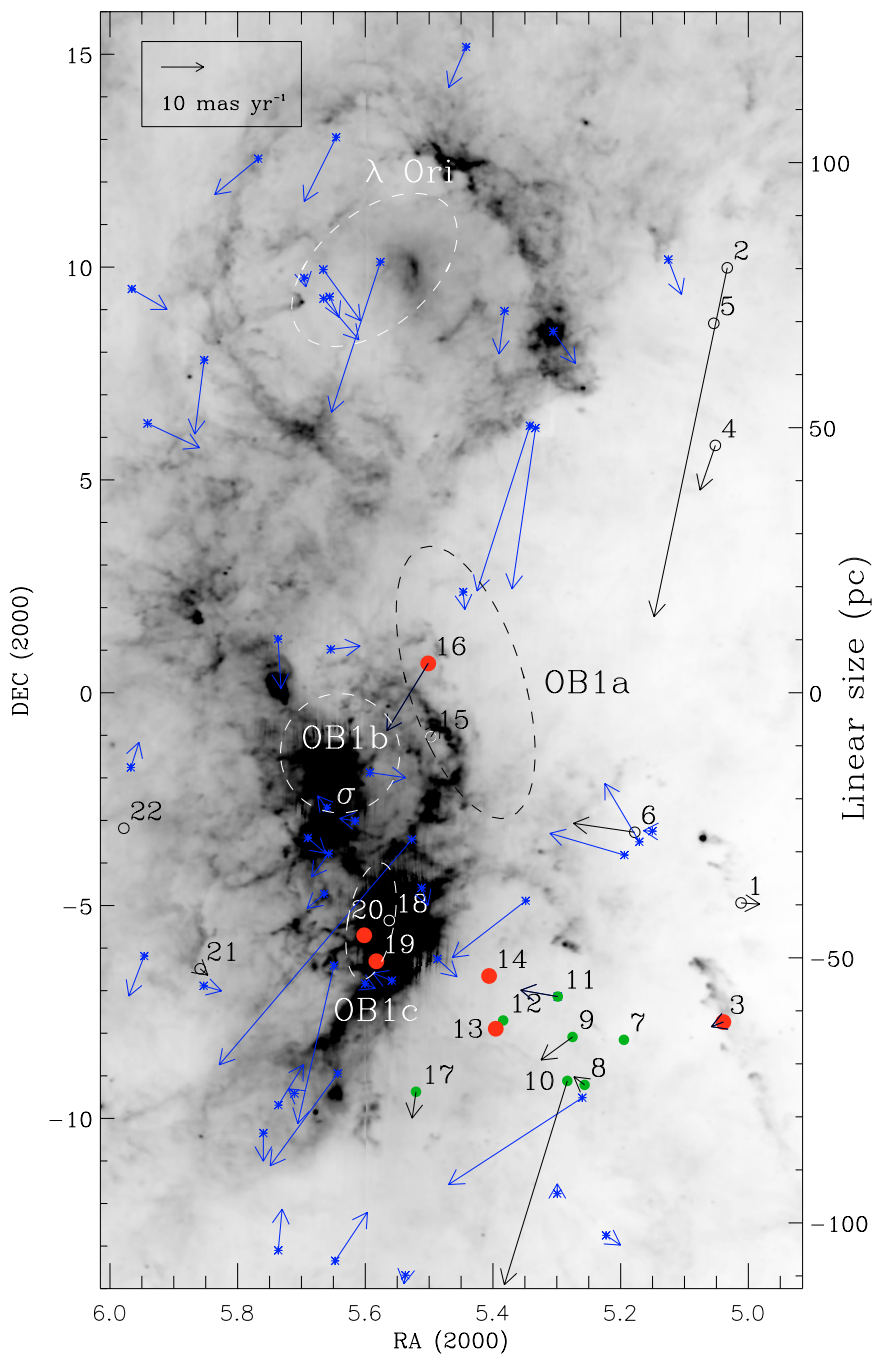

Fig. 10. Spatial distribution of the stars in our sample (big dots for bona-fide PMS stars, small dots for stars with lithium detected, open circles for stars with undetectable lithium) and of the RASS PMS stars (asterisks) of Alcalá et al. (2000). The points are labeled according to the code given in Tables 1, 3 and 4 . The arrows represent the propermotion vectors. The grey-scale image in the background is the $100 \mu \mathrm{m}$ IRAS flux map. The Blaauw's cluster Ori OB1a-b-c, and $\lambda$ Ori region are also marked with dashed contours. The right-side y-axis shows the image scale in parsecs at the mean distance of the Orion complex $(460 \mathrm{pc})$.

RX J0502.0+0959 in the HR diagram is consistent with its main sequence nature.

Among the six stars with lithium strength above the Pleiades upper envelope in the Li equivalent width versus color diagram, shown in Fig. 9, two were previously identified by Alcalá et al. (2000). This gives support to the conclusion that the other four stars are also in the T Tauri evolutionary status.

The fact that the stars in our sample are spread over several degrees in the general direction of Orion and the assumption of the same distance of $460 \mathrm{pc}$ for all of them, may have strong consequences on the derived stellar properties. In particular, stars actually located at a distance less than $460 \mathrm{pc}$, will have overestimated luminosities and radii. The ages of these stars

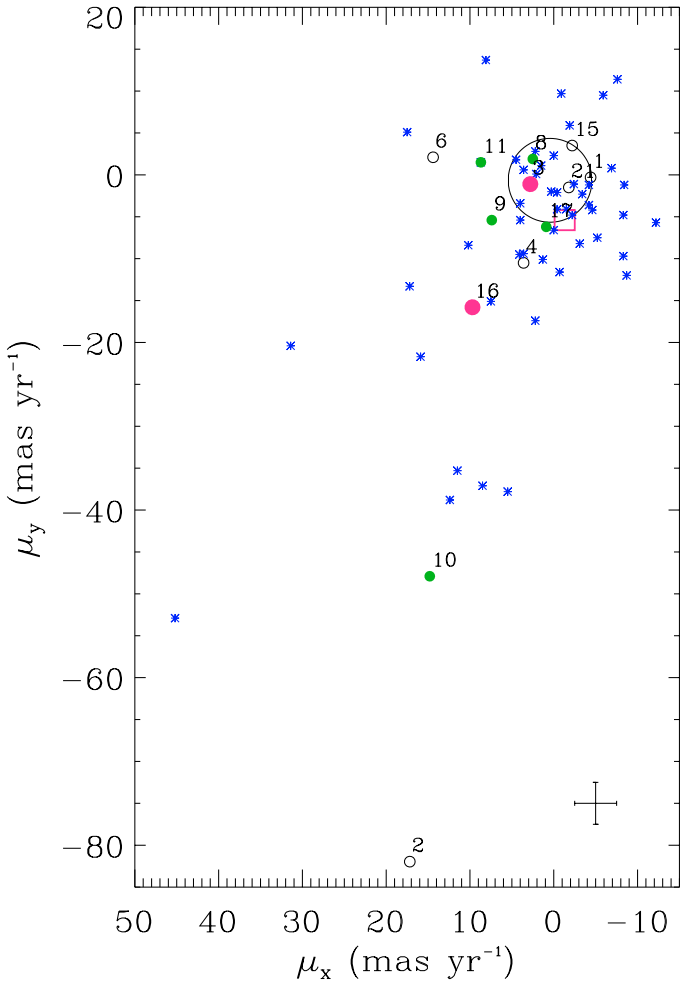

Fig. 11. Proper motions of the stars in our sample (filled and open circles, as in Fig. 10) and of the RASS PMS stars (asterisks) of Alcalá et al. (2000). The points are labeled according to the code given in Tables 1, 3 and 4. Error bars typical of Thyco proper motions are shown in the lower right corner. The open square marks the "central coordinates" of the proper motion distribution, while the big circle defines the outer limit given by de Zeeuw et al. (1999) for membership to the Ori OB1 association.

will be underestimated and, for stars earlier than about K5, the masses will be overestimated.

It is indeed interesting to note that, assuming the same distance of $460 \mathrm{pc}$ for all the lithium stars, three stars with strong lithium (1RXS J052345.0-075343, RX J0524.4-0640 and RX J0530.1+0041) result younger than $2 \mathrm{Myr}$, but lie some 10-20 pc away from the main Orion molecular clouds, while other two (RX J0535.0-0618 and RX J0536.1-0541) lie superposed on the Orion A molecular cloud and are 5-10 Myr old. The latter may be stars with motions towards the Sun. Many more similar stars should exist alongside and behind the clouds if dispersal is isotropic.

For the very young dispersed PMS stars, in addition to the scenario of star formation in small turbulent cloudlets proposed by Feigelson (1996), other authors (Sterzik \& Durisen 1995) suggested that, due to three body interactions in young multiple systems, some stars may acquire a higher velocity dispersion than the canonical one of $1 \mathrm{~km} \mathrm{~s}^{-1}$ observed in SFRs (Lada $\&$ Lada 1991). However, such ejection mechanism is efficient only for very low-mass stars.

In the case of $\mathrm{OB}$ associations, star formation may be triggered by compression of clouds due to the radiation and winds of OB stars, or supernova shock waves. A consequence of this process is that star formation is sequential. Triggered star formation is taking place in the Orion $\mathrm{OB}$ association, where 


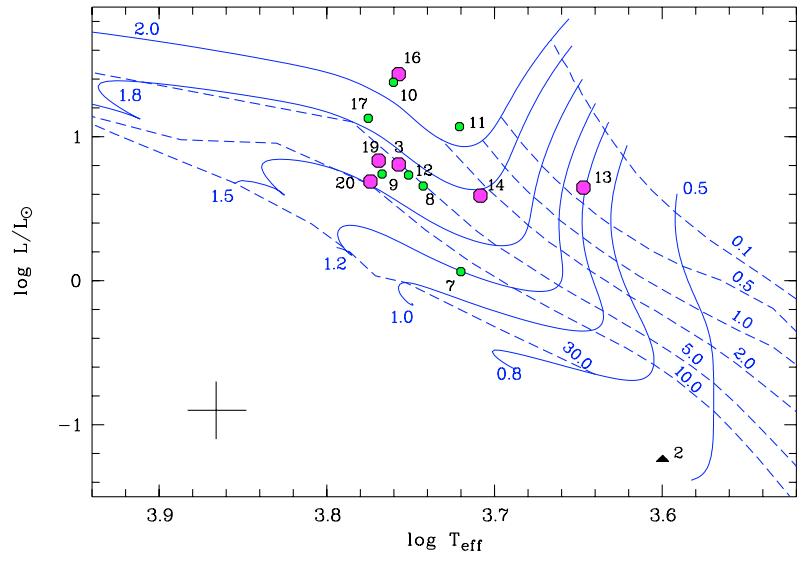

Fig. 12. HR diagram of the stars with lithium in our sample. The meaning of the symbols is the same as in Figs. 10 and 11. The theoretical PMS evolutionary tracks by D'Antona and Mazzitelli (1997) are overplotted for masses in the range from 0.5 to $2 M_{\odot}$ (continuous lines) and ages from 0.1 to $30.0 \mathrm{Myr}$. (dashed lines). The points are labelled according to the code given in Tables 1, 3 and 4. The star RX J0502.0+0959, the only object for which a reliable parallax is available, is indicated with the black triangle. The average errors are also indicated.

several cometary clouds are observed (Ogura \& Sugitani 1998). Also, evidence for sequential star formation has recently been observed in the $\lambda$ Orionis region (Dolan \& Mathieu 2002) and in the L1616 cometary cloud (Stanke et al. 2002; Alcalá et al., in preparation) to the south-west of the Orion A cloud. Moreover, several strong lithium stars have been identified spread between the south-west of the Orion A cloud and some of the cometary clouds in Orion (Alcalá et al. 2000). The strong lithium stars in our sample that lie far from molecular clouds provide more evidence for the spread population of young stars in Orion, probably formed by triggered star formation.

\section{Conclusions}

Further identifications of ROSAT X-ray sources distributed in the general direction of the Orion star forming region were presented. The identification of the X-ray sources was based on medium-resolution optical spectroscopy and by $U B V$ Johnson photoelectric photometry using a $1 \mathrm{~m}$-class telescope. For this reason the sample was limited to $22 \mathrm{X}$-rays sources and the most likely optical counterparts are brighter than $V=12$ mag. Despite of this, the lithium absorption line could be detected in the spectra of thirteen stars which are good candidates for low-mass PMS stars: six of the lithium-rich stars can be classified as bona-fide WTTS, with four of them, namely 1RXS J052345.0-075343, RX J0524.4-0640, RX J0535.00618 and RX J0536.1-0541, being new identifications, while other five stars have a lithium strength consistent with the maximum observed for the Pleiades and hence, are good candidates for WTTS. Broad and, in a few cases, asymmetric $\mathrm{H} \alpha$ residual profiles, indicative of accretion, have been observed in several objects.

The stellar parameters for the new WTTS and PMS candidates in the sample were determined adopting a distance of
$460 \mathrm{pc}$ and the comparison of the position of these stars in the HR diagram with the theoretical PMS evolutionary tracks by D'Antona \& Mazzitelli (1997) yields masses in the range from 0.8 to $2 M_{\odot}$ and ages younger than $10 \mathrm{Myr}$.

The kinematical study, based on proper motions extracted from the Tycho catalogue, shows that the proper motions of the present sample are consistent with those of the pre-main sequence stars selected by Alcalá et al. (2000), which, at the same time, are consistent with those of stars belonging to the Orion Nebula Cluster and to the hot stars of the Ori OB1 association. This gives further support for the membership of these samples to the Orion complex.

Three groups of PMS stars with coherent proper motions were identified and coincide in position with two of the X-ray clumps predicted by the statistical studies of the large scale spatial distribution of RASS X-ray sources in Orion by Sterzik et al. (1995). With the present data we cannot draw any conclusion on the possible link between these clumps and the cloudlets predicted in the simulations by Feigelson (1996). However, these relatively isolated and compact regions, which presumably harbour new SFRs, are suitable to conduct a complete search for new low-mass PMS stars and determination of their initial mass function, and will be the subject of forthcoming work.

Acknowledgements. This work has been supported by the Agenzia Spaziale Italiana (contract No. I/R/111/01) and by the Regione Sicilia which are gratefully acknowledged. This research has made use of the NASA/IPAC Infrared Science Archive, who is operated by the Jet Propulsion Laboratory, California Institute of Technology, under contract with the National Aeronautics and Space Administration. We would like to thank the referee for very useful comments and suggestions. This research has made use of the SIMBAD and VIZIER databases, operated at CDS, Strasbourg, France. The ROSAT project has been supported by the Bundesministerium für Forschung und Technologie and the Max-Planck-Gesellschaft.

\section{References}

Alcalá, J. M., Terranegra, L., Wichmann, R., Chavarria, C., \& Krautter, J. 1996, A\&AS, 119, 7

Alcalá, J. M., Covino, E., Torres, G., et al. 2000, A\&A, 353, 186

Amado, P. J., \& Byrne, P. B. 1997, A\&A, 319, 967

Bailey, J. 1998, MNRAS, 301, 161

Blaauw, A. 1991, in The Physics of Star Formation and Early Stellar Evolution, ed. C. J. Lada, \& N. D. Kylafis (Kluwer Academic Publishers), 125

Bertout, C., Robichon, N., \& Arenou, F. 1999, A\&A, 352, 574

Carpenter, J. M., \& Lynne, A. H. 2001, AJ, 121, 3160

Covino, E., Alcalá, J. M., Allain, S., et al. 1997, A\&A, 328, 187

Cox, A. N. 2000, Allen's Astrophysical Quantities (Springer-Verlag)

Crawford, D. L., \& Mandwewala, N. 1976, PASP, 88, 917

D’Antona, F., \& Mazzitelli, I. 1997, Mem. Soc. Astron. It., 68, 807

Dolan, C. J., \& Mathieu, R. D. 2001, AJ, 121, 2124

Dolan, C. J., \& Mathieu R. D. 2002, AJ, 123, 387

de Zeeuw, P. T., Hoogerwerf, R., de Bruijne, J. H. J., Brown, A. J. A., \& Blaauw, A. 1999, ApJ, 117, 354

Duflot, M., Figon, P., \& Meyssonnier, N. 1995, A\&AS, 114, 269

Evans, D. S. 1967, in IAU Symp. 30, ed. A. H. Batten, \& J. F. Heard (Academic Press, London), 57 
Feigelson, E. D. 1996, ApJ, 468, 306

Feigelson, E. D., \& Montmerle, T. 1999, ARA\&A, 37, 363

FitzGerald, M. P. 1970, A\&A, 4, 234

Flower, P. J. ApJ, 469, 355

Frasca, A., \& Catalano, S. 1994, A\&A, 284, 883

Frasca, A., Freire Ferrero, R., Marilli, E., \& Catalano, S. 2000, A\&A, 364,179

Frink, S., Roeser, S., Alcalá, J. M., Covino, E., \& Brandner, W. 1998, A\&A, 338, 442

Genzel, R., \& Stutski, J. 1989, ARA\&A, 27, 41

Guilliout, P., Sterzik, M. F., Schmitt, J. H. M. M., Motch, C., \& Neuhäuser, R. 1998, A\&A, 337, 113

ESA 1997, The Hipparcos Catalogue, ESA SP-1200

Hog, E., Fabricius, C., \& Makarov, V. V., et al. 2000, A\&A, 355, L27

Jones, B. F., \& Walker, M. F. 1988, AJ, 95, 1755

Lada, C. J., \& Lada, E. A. 1991, in The formation and evolution of stars clusters, ed. K. Janes, p. 3

Landolt, A. U. 1992, AJ, 104, 340

Lo Presti, C., \& Marilli, E. 1993, PHOT. Photometrical Data Reduction Package. Internal report of Catania Astrophysical Observatory N. 2/1993

McNamara, B. J., \& Huels, S. 1983, A\&AS, 54, 221

McNamara, B. J., Hack, W. J., Olson, R. W., \& Mathieu, R. D. 1989, AJ, 97, 1427

Maddalena, R. J., Morris, M., Moscowitz, J., \& Thaddeus, P. 1986, ApJ, 303, 375

Martín, E. L. 1997, A\&A, 321, 492

Martín, E. L., \& Magazzù, A. 1999, A\&A, 342, 173

Mamajek, E. E., Meyer, M. R., \& Liebert, J. W. 2002, AJ, 124, 1670

Monet, D. G., Levine, S. E., Casian, B., et al. 2003, AJ, 125, 984
Mermilliod, J. C., Mermilliod, M., \& Hauck, B. 1997, A\&AS, 124, 349

Neuhäuser, R., Sterzik, M. F., Schmitt, J. H. M. M., Wichmann, R., \& Krautter, J. 1995, A\&A, 297, 391

Ogura, K., \& Sugitani, K. 1998, PASA, 15, 91

Parenago, P. P. 1954, Trudy Sternberg Astr. Inst. vol. 25

Preibisch, T., Günter, E., Zinnecker, H., et al. 1998, A\&A, 333, 619

Stanke, T., Smith, M. D., Gredel, R., \& Szokoly, G. 2002, A\&A, 393, 251

Sterzik, M. F., \& Durisen, R. H. 1995, A\&A, 304, 9

Sterzik, M., Alcalá, J. M., Neuhaeuser, R., \& Schmitt, J. H. M. M. 1995, A\&A, 297, 418

Soderblom, D. R., Jones, B. F., Balachandran, S., Stauffer, J. R., Duncan, D. K., et al. 1993, AJ, 106, 1059

Takami, M., Bailey, J., \& Chrysostomou, A. 2003, A\&A, 397, 675

Tian, K. P., van Leeuwen, F., Zhao, J. L., \& Su, C. G. 1996, A\&AS, 118,503

Tonry, J., \& Davis, M. 1979, AJ, 84, 1511

van Altena, W. F., Lee, J. T., Lee, J.-F., Lu, P. K., \& Upgren, A. R. 1988, AJ, 95, 1744

Walker, M. F. 1983, ApJ, 271, 642

Walter, F. M. 1986, ApJ, 306, 573

Walter, F. M., Alcalá, J. M., Neuhauser, R., Sterzik, M., \& Wolk, S. J. 2000, Protostars and Planets IV, University of Arizona Press, ed. V. Mannings, A. P. Boss, \& S. S. Russell, p. 273

Wichmann, R., Krautter, J., Covino, E., Alcalá, J. M., Neuhäuser, R., \& Schmitt, J. H. M. M. 1997, A\&A, 320, 185

Wichmann, R., Covino, E., Alcalá, J. M., et al. 1999, MNRAS, 307, 909 